\title{
Cold roll forming of a U-channel made of high strength steel
}

\author{
M. Lindgren \\ Dalarna University, Sweden \\ Received 16 June 2005; received in revised form 3 November 2006; accepted 6 December 2006
}

\begin{abstract}
Cold roll forming is a bending process where the bending occurs gradually in several forming steps from an undeformed strip to a finished profile. The process is very interesting for the sheet metal industry due to the high speed in which the profile can be produced. High strength steel has, in recent years, become more common in cold roll forming. These materials have advantages but also disadvantages that affect the design of the process.

Simple models in literature [K.F. Chiang, Cold roll forming, ME Thesis, University of Auckland, August 1984] predict that the longitudinal peak membrane strain in the flange of a profile is independent of the material properties. However, Ingvarsson [L. Ingvarsson, Förenklad teori för rullforming av elementär v-profil, jämförelse mellan normalt och höghållfast stål, VAMP 15- rullforming 23 april 2001 ] compared mild and ultra high strength in a roll forming experiment and the conclusion was that the material properties will affect the finished profile. This paper is a fundamental study performed in order to understand the observation by Ingvarsson [L. Ingvarsson, Förenklad teori för rullforming av elementär v-profil, jämförelse mellan normalt och höghållfast stål, VAMP 15- rullforming 23 april 2001].

The objectives of this study are to investigate the change in the longitudinal peak membrane strain at the flange edge and the deformation length when the yield strength increases. These are important since they can be used to determine the number of forming steps and the distance between them when designing the cold roll forming machine. The result from the simulations show that the longitudinal peak membrane strain decreases and the deformation length increases when the yield strength is increased.
\end{abstract}

(C) 2007 Published by Elsevier B.V.

Keywords: Cold roll forming; High strength steel; Finite element analysis

\section{Introduction}

In cold roll forming a profile is formed in several forming steps from an undeformed strip to a finished profile (Fig. 1). The forming process is geometrically complicated due to the fact that the forming does not only occur in the tools but also between each forming stand. When creating the tools the tool designer must decide how many forming steps the profile demands. The number of steps is dependent on the shape of the cross-section, tolerance, thickness and the material properties.

It is important to minimise the number of steps as this reduces the cost of the cold roll forming machine. Then the process can be a competitive alternative also for smaller production volumes. Therefore the knowledge of how high strength steel affects the number of forming steps is important.

Existing relations between the longitudinal peak membrane strain, deformation length and the yield strength of the mate-

E-mail address: mlg@du.se. rial have been investigated and compared with finite element analysis in this study.

\section{Notations}

The notations are given below and in Fig. 2:

- $L$, deformation length;

- $a$, flange length;

- $t$, thickness of the strip;

- $Y$, bend angle;

- $r$, distance from the bending zone;

- $z$, distance from where the bending starts;

- $e$, the longitudinal membrane engineering strain at the flange.

\section{Background}

The profile is formed in several steps and that will cause longitudinal strain in the flange. The strain develops as the material in a flange of a profile will travel a longer distance than the 


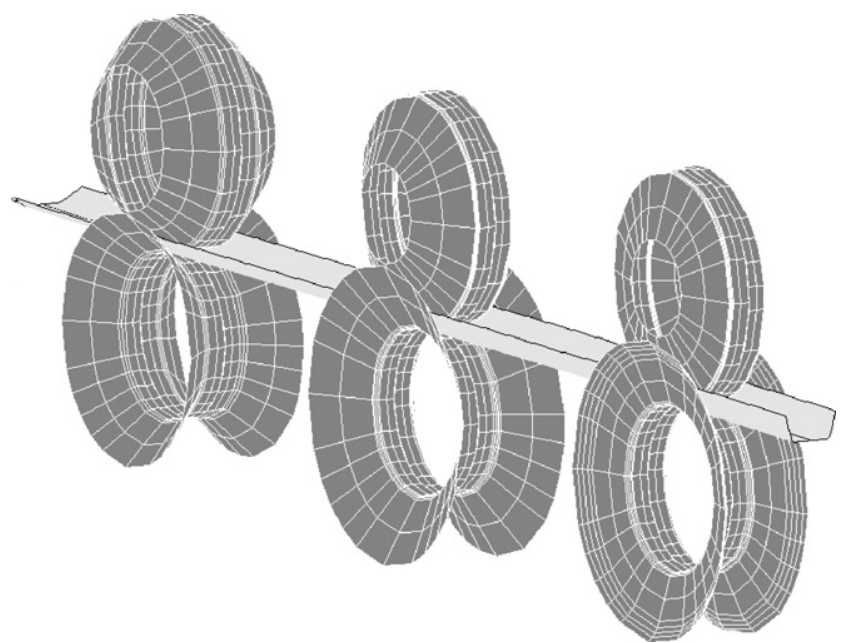

Fig. 1. The profile is formed in several forming stands from an undeformed strip to a finished profile.

material in the bending zone. Panton et al. [1] concluded that the longitudinal peak strain occurs when the strip is in contact with the rolls for the first time. The peak strain should not be plastic as plastic strain will give a residual stress that causes defects on the profile as wave edges, longitudinal curvature, etc.

Bhattacharyya et al. [2] created a model of the deformation length by minimising the total plastic work for a U-channel. The obtained model predicts that the deformation length is independent of yield strength. It is written as:

$L=\sqrt{\frac{8 a^{3} \theta}{3 t}}$
Chiang [3] derived a model for the longitudinal engineering strain in the flange based on minimising of the plastic work due to stretching and bending of the profile. It is written as:

$\left.e=\frac{9}{32}\left(\frac{t^{2}}{a^{6}}\right) r^{2} z^{2}\right\}_{0<z<L}^{0<r<a}$

The model overestimated the strain when the strip approached the tool. Therefore Chiang derived an improved expression by a geometrical consideration for the peak strain, leading to:

$e=\sqrt{1+2\left(\frac{a}{L}\right)^{2}(1-\cos \theta)}-1$

This model showed that the longitudinal strain is uniform in the deformation zone. All models predict that the behaviour is independent of the material properties.

Ingvarsson [4] compared mild steel with ultra high strength steel in an experiment where a V-section was cold roll formed with the same number of forming stands. The ultra high strength steel gave a straight profile, but not the mild steel. The conclusion was that fewer forming stands could be used when ultra high strength steel is being roll formed.

\section{Approach}

Finite element simulations are used in the current study to evaluate the yield strength influence on peak strain and deformation length. Since the analytical formulas for longitudinal peak membrane strain do not account for the yield strength.

Several papers have been written about the finite element simulation on cold roll forming for example [5-7].

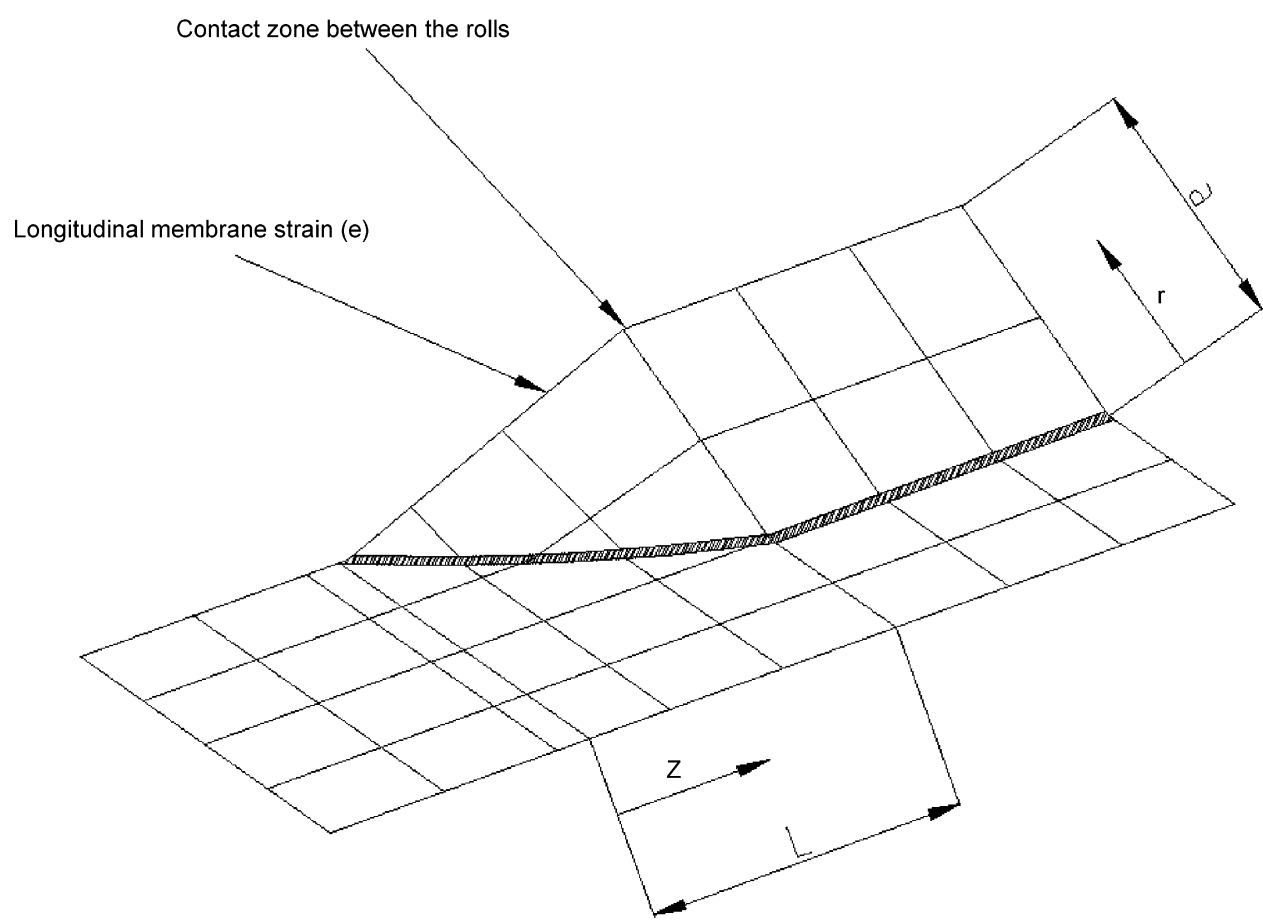

Fig. 2. One half of the U-channel. The strip is formed by a bend angle (Y8) in the contact zone between the rolls. It will cause a longitudinal membrane strain (e) in the flange. 
Table 1

Series of experiments where the forming steps are 08 (feeder roll)-108-108 and 08 (feeder roll)-208-208, when seven different yield strengths are used

\begin{tabular}{lll}
\hline & $08-108-108$ & $08-208-208$ \\
\hline $200 \mathrm{MPa}$ & $\times$ & $\times$ \\
$400 \mathrm{MPa}$ & $\times$ & $\times$ \\
$600 \mathrm{MPa}$ & $\times$ & $\times$ \\
$800 \mathrm{MPa}$ & $\times$ & $\times$ \\
$1000 \mathrm{MPa}$ & $\times$ & $\times$ \\
$1200 \mathrm{MPa}$ & $\times$ & $\times$ \\
$1400 \mathrm{MPa}$ & $\times \times$ & $\times$ \\
\hline
\end{tabular}

\section{The finite element model}

Fourteen different simulations have been carried out with varying yield strengths and bend angles (Table 1). The finite element package MARC/MENTAT was used to perform the simulations.

The simulation starts from an undeformed strip and stops when the material reaches the second forming step. The total-, plastic- and elastic longitudinal peak membrane strain at the edge of the flange, are evaluated.

\subsection{The geometry}

The model consists of four forming stands where the first two stands are used as a belt feeder to the others (Fig. 3). The length of the strip is $800 \mathrm{~mm}$ so that the forming steps 08 (feeder rolls)-108-108 and 08 (feeder rolls)-208-208 are engaged at the same time when the simulation has come to an end. An evaluation of the longitudinal peak membrane strain and the deformation length is then performed for the first forming step where bending occurs.

The cross-section geometry of the strip is, width $40 \mathrm{~mm}$, flange length $(a) 10 \mathrm{~mm}$, bending radius $3 \mathrm{~mm}$ and the thickness $(t)$ of the strip is $1.5 \mathrm{~mm}$. Due to the symmetry only half of the strip is modelled.

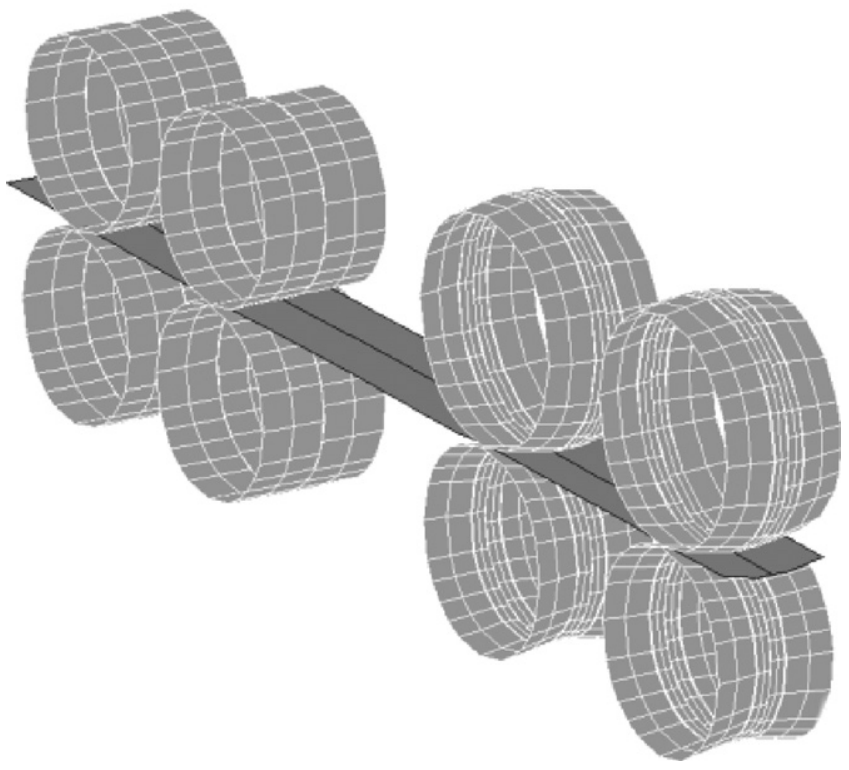

Fig. 3. Four forming stands are used. The two first stands are used as a belt feeder. The other two forming stands have the same bend angle $(Y)$.

The strip is modelled with 1600 thick shell elements. The mesh size is $4 \mathrm{~mm} \times 1 \mathrm{~mm}$ in the bending zone and in other regions the mesh size is $4 \mathrm{~mm} \times 4 \mathrm{~mm}$.

The strip is modelled with a bilinear thick shell element type number 75 [8]. This is a four-node element that calculates the membrane strain in the middle surface. Three layers of integration points are used in the thickness direction.

The rolls are modelled as rigid surfaces and they rotate, giving the strip an initial speed of $0.6 \mathrm{~m} / \mathrm{s}$. The speed is then increased by $0.5 \%$ in each forming stand to counteract buckling.

\subsection{Contact}

The clearance between the tools is $1.5 \mathrm{~mm}$ and the strip thickness is $1.504 \mathrm{~mm}$, which gives a contact pressure of $560 \mathrm{MPa}$. The friction is modelled as Coulomb friction and the friction coefficient is 0.1 .

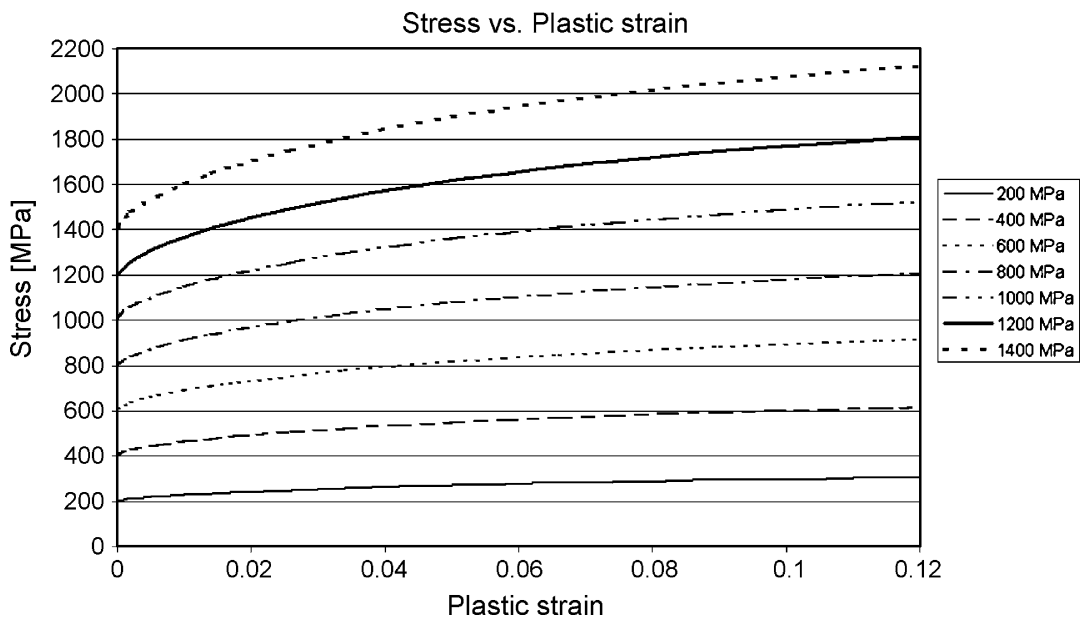

Fig. 4. The fictive tensile test data for seven different materials. They have been implemented in the finite element program as a table. 


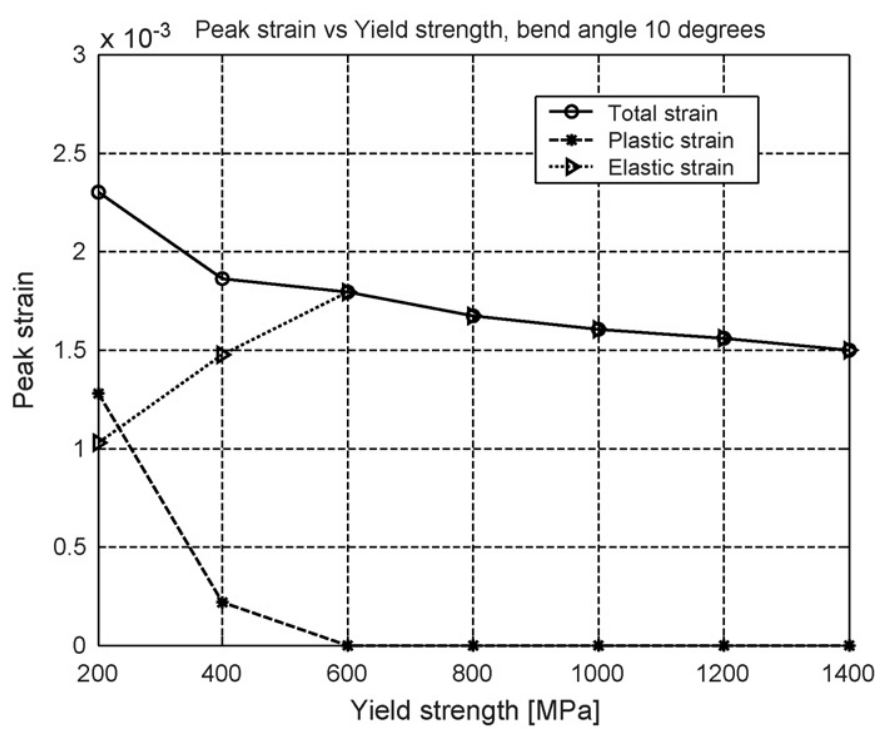

Fig. 5. When the virgin yield strength increases the longitudinal peak membrane strain will decrease. When the plastic strain goes to zero the total strain will flatten out and the strain is purely elastic.

\subsection{Material model}

The material is modelled as an elasto-plastic material. The material hardening is isotropic and the von Mises yield surface and the associated flow rule are used. A fictive tensile test data is implemented in the finite element program as a table. The yield strength is then scaled so the material starts to yield in seven different points from 200 to $1400 \mathrm{MPa}$ (Fig. 4). The model account for large deformations and strains. An additive decomposition of total strain rate into elastic and plastic strain rates is assumed.

\section{Result and discussion}

Fig. 5 shows the peak longitudinal membrane strain of the edge due to bending from an originally flat strip to a 108 angle for the different simulations. The result for each simulation is denoted by the virgin yield strength of the flow stress in Fig. 4 used in respective simulation. This value is given on the horizontal axis in Fig. 5. The simulations show for a bend angle of 108 that the total (plastic and elastic) strain decreases when the virgin yield strength increases. The total strain decreases more in the beginning when plastic strain is present.

Fig. 6 is the bend angle 208 and the behaviour is similar as in Fig. 5. The difference between the cases is that the strain becomes purely elastic at higher material virgin yield strength. The strain is also greater for a bending angle of 208, which agrees with Chiang [3].

The deformation length, Eq. (1), is the distance between where the transverse bending starts and the forming stands. In this study a strain based deformation length is used. It is defined as the distance from the forming stand to the point in the flange edge where the strain is greater than $2 e-5$. The strain based deformation length for the cases 108 and 208 will increase when the yield strength increases (Fig. 7). The length is greater for the

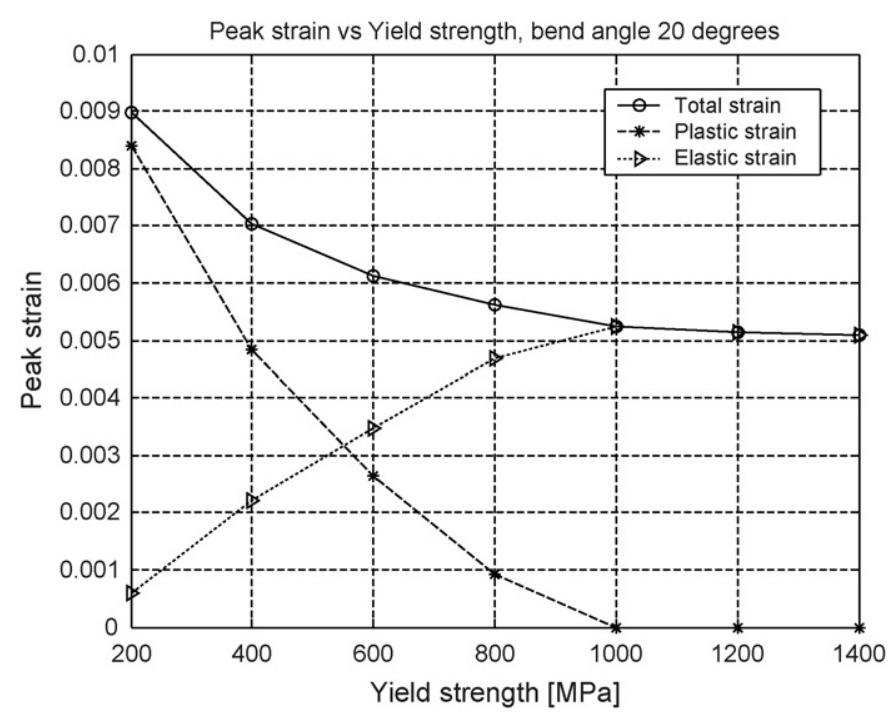

Fig. 6. The behaviour for the longitudinal peak membrane strain is similar to the case with a forming step of 108 . But now is the curve for the elastic and plastic longitudinal peak membrane strain displaced to a higher level of virgin yield strength.

bend angle 208 than 108, which agrees with Bhattacharyya et al. [2].

Figs. 7 and 8 are the simulation results for the peak strain and the deformation length compared with the models, Eqs. (1) and (3), that Bhattacharyya et al. and Chiang derived. One can see that they do not agree due to the large simplifications in the assumed pattern and material behaviour in their models [2,3]. The assumptions for the deformation length were:

- The material is rigid perfectly plastic.

- Bending takes place only along the fold-line.

- The longitudinal bending of the web and the out-of-plane bending of the flange can be neglected.

- The flange adopts the shape that minimises the plastic work.

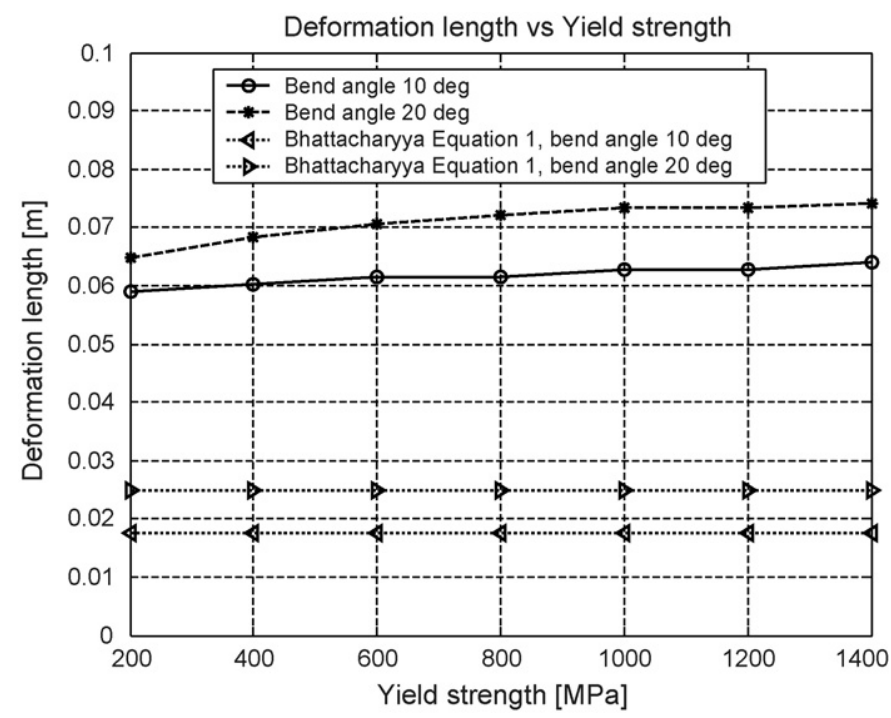

Fig. 7. The deformation length for bend angle 108 and 208 will increase when the yield strength increases. The length is greater for bend angle 208 . 


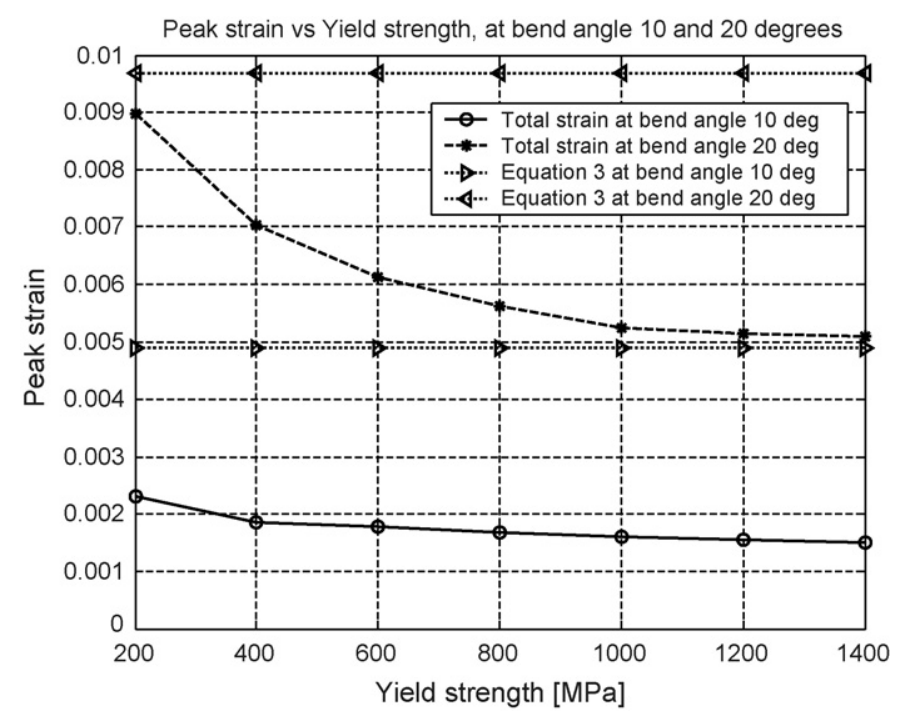

Fig. 8. The simulations are compared with the model Chiang derived, Eq. (3), and one can see that they do not agree well.

The model for the peak strain, Eq. (3), has the additional assumption that the flange edge remains straight during the deformation.

\section{Conclusions}

The simulations show that the longitudinal peak membrane strain decreases, the deformation length increases for materials with higher yield strength. This information has not been possible to obtain from simple models as in Eqs. (1)-(3).

Decreasing longitudinal peak membrane strain gives less residual stresses in the flange of the profile and quality problems as wave edges, longitudinal curvature, end flare, etc. will decrease. It will make it possible to use fewer forming steps for profiles made of high strength steel. However, high strength steel has larger spring back that has to be accounted for. This is the explanation why Ingvarsson [4] obtained a straight V-profile when ultra high strength steel was used. Ingvarsson [4] used the same number of forming steps for both the mild and the ultra high strength steel. For mild steels were the forming steps too few and larger residual stresses were present after the forming. The latter gave a V-profile with a longitudinal curvature.

Increasing deformation length leads to the horizontal distance between the forming steps must increase when high strength steel is used.

\section{Acknowledgements}

The author thanks ORTIC AB, Swedish Knowledge Foundation, Jernkontoret and Dalarna University for their technical and financial support.

\section{References}

[1] S.M. Panton, S.D. Zhu, J.L. Duncan, Geometric constraints on the forming path in roll forming channel sections, Proc. Inst. Mech. Eng. 206.

[2] D. Bhattacharyya, P.D. Smith, C.H. Yee, L.F. Collins, The prediction of deformation length in cold roll forming, J. Mech. Work. Tech. 9 (1984) 181-191.

[3] K.F. Chiang, Cold roll forming, ME Thesis, University of Auckland, August 1984.

[4] L. Ingvarsson, Förenklad teori för rullforming av elementär v-profil, jämförelse mellan normalt och höghållfast stål, VAMP 15- rullforming 23 april 2001.

[5] M. Brunet, S. Mguil, P. Pol, Modelling of a roll-forming process with a combined 2D and 3D FEM code, J. Mater. Process. Technol. 80-81 (1998) 213-219.

[6] M. Farzin, M.S. Tehrani, E. Shameli, Determination of buckling limit of strain in cold roll forming by finite element analysis, J. Mater. Process. Technol. 125-126 (2002) 626-632.

[7] M. Lindgren, Finite element model of roll forming of a U-channel profile, To be presented at International Conference on Techn. of Plasticity, Verona, 2005.

[8] MARC, Element Library, vol. B, Marc Analysis Research Corporation, USA. 